\title{
QUASAR ACTIVITY IN RICH GALAXY CLUSTERS
}

\author{
H. K. C. Yee \\ Department of Astronomy, University of Toronto, Toronto, Ont. M5S \\ 1A6, Canada \\ E. Ellingson \\ Dominion Astrophysical Observatory, Victoria, BC, V8X 4M6, Canada
}

We have carried out a number of imaging surveys of fields around quasars to study their global environments (e.g. Yee and Green 1987, Ellingson, Yee and Green 1991). The richness of the galaxy cluster environment of each quasar was determined using the galaxy-quasar spatial covariance amplitude, a quantity which is normalized for the expected luminosity and spatial distribution of galaxies (Longair and Seldner 1978, Yee and Green 1987). We find that 40\% of the brightest radio-loud quasars inhabit rich clusters of galaxies (Abell class 1 or higher) at $z \gtrless 0.5$ whereas only fainter AGN inhabit clusters at more recent epochs (Figure 1). This can be understood if quasars in rich clusters evolve much faster than those in poor environments.

Where are the remnants of these once bright quasars that were the central objects in rich clusters? FR class I radio galaxies are often the brightest members of nearby rich clusters. High signal-to-noise ratio spectroscopy shows that these objects often have a faint quasar-like nuclear spectrum (DeRobertis and Yee 1990). In Fig. 1 we include a sample of 3CR radio galaxies with $z<0.1$, showing that their nuclear luminosities may be consistent with the extrapolation of the quasar data.

In order to analyse this evolution quantitatively, taking into account the luminosity function (LF) of active objects and volume selection effects, we construct "envelope models" on the $M_{q s 0^{-}} z$ plane. Given a LF and its evolution, one can predict the magnitude of the brightest quasar which one would expect statistically to see at a given redshift. In Fig. 1, the dotted line represents the envelope expected for the quasar LF and evolution derived by Boyle et al. (1987) for optically-selected quasars. This model matches the upper envelope of both quasars and radio galaxies well, suggesting that the LF for optically-selected quasars is also a reasonable fit for radio-loud quasars, and that radio galaxies and radio-loud quasars can be described by a single LF and evolution model. Also plotted in Figure 1 is a model which describes the upper envelope of AGN in rich environments, where evolution 5 to 6 times faster is required.

The data presented provide forceful evidence that the evolution of quasar activity is a stong function of their environment. One possible reason is an increase in the cluster core velocity dispersion decreases the efficiency of galaxy interactions (e.g. Roos 1985, DeRobertis 1985). Alternatively, the rapid evolution of quasars in clusters may be due to an increase of the intracluster 
gas which produces a more efficient stripping of the host or companions (Stocke and Perrenod 1981).

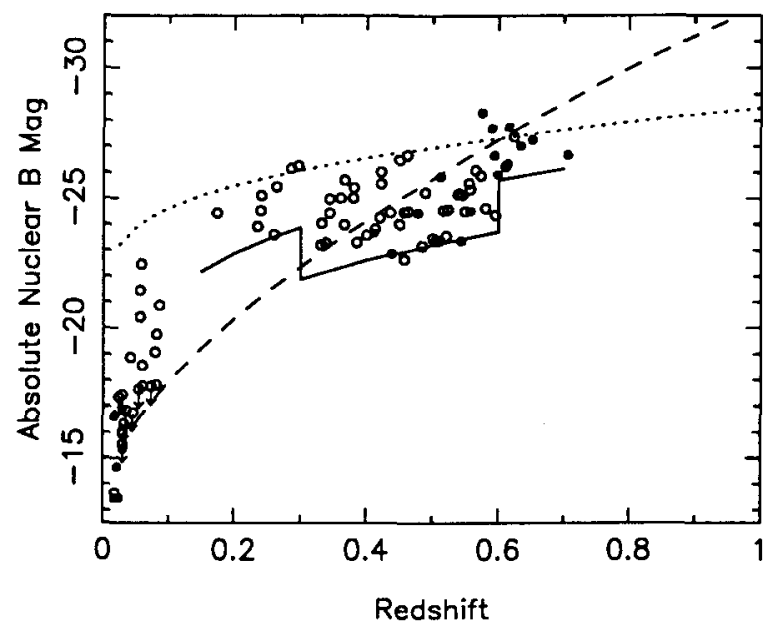

Figure 1. Plot of $M_{q s o}$ vs $z$. Objects situated in rich environments (Abell class 1 or richer) are plotted as solid dots, while those in poorer environments are shown as open dots. The solid line represents the redshift and apparent magnitude sample limits of the quasar sample. Objects with $z>0.15$ are from the quasar samples while those in the radio galaxy sample have $z<0.1$. The dotted line represents the envelope model predicted by the Boyle et al. (1987) quasar LF and evolution, while the dashed line represents evolution 5 to 6 times faster.

To test the first scenario, we are carrying out a program of spectroscopic observations of faint galaxies in the fields of quasars (Ellingson, Green and Yee 1991, Ellingson and Yee 1992, in preparation). To date, relative velocities of more than 130 galaxies associated with quasars have been determined. Preliminary analysis shows that the velocity dispersions of clusters associated with bright quasars at high redshift $(z z 0.5)$ are significantly lower than normal Abell clusters at low redshift, $\left(\sim 500 v s \sim 800 \mathrm{~km} \mathrm{~s}^{-1}\right)$, in agreement with the models discussed above. Clusters associated with fainter quasars are found to have dispersions intermediate between the two. These results strongly suggest that clusters hosting bright quasars are in relatively young dynamical states, and that the rapid fading of the AGN nucleus in these environments is linked to the dynamical evolution of the cluster core.

\section{REFERENCES}

Boyle, B. J. et al. 1987, M. N. R. A. S., 227, 717.

De Robertis, M. M. 1985, A. J., 235, 351.

De Robertis, M. M. and Yee, H. K. C. 1990, A. J., 100, 84.

Ellingson, E., Green, R. F. and Yee, H. K. C. 1991, Ap. J., 378, 476.

Ellingson, E., Yee, H. K. C., and Green, R. F. 1991, Ap. J., 371, 49.

Longair, M. S. and Seldner, M. 1979, M. N. R. A. S., 189, 433.

Roos, N. 1985, A. A., 104, 218.

Stocke, J. T. and Perrenod, S. C. 1981, Ap. J., 245, 375.

Yee, H. K. C. and Green, R. F. 1987, Ap. J., 319, 28. 